\title{
Theory on another type of temperature-independent superconductivity based on circuit approaches with high critical current density
}

\author{
Shinichi Ishiguri \\ Nihon University \\ 1-2-1 Izumi-Cho, Narashinoshi, Chiba 275-8575 JAPAN \\ TEL: +81-47-474-9143 \\ Email: ishiguri.shinichi@nihon-u.ac.jp
}

\begin{abstract}
This paper proposes a method to generate a new type of superconductivity that is temperature independent with a high critical current density. This study is significant because the method does not require refrigeration, specific setups, or specific substances. That is, the method for generating the superconductivity is very simple. Many conventional superconductor studies have not yet reached this point. Moreover, compared with our previously developed superconductivity (PNS) [1-3], the critical currents in this study are much larger, which is important for practical applications. In the theoretical approaches, even though the mechanism of pairing, and the Bose-Einstein condensation are the same in this study as in PNS, the present paper emphasizes the mechanism of the Meissner effect in addition to formulating the critical current density. Further, we establish a simulation method with an equivalent circuit that reveals the superconductivity properties in terms of the transport current and the electromagnetic characteristics.

The principles of the presented system are as follows:

1. First a voltage source, a current source and a load are connected in series.

2. Then, the voltage of the voltage source is adjusted to balance the voltage of the load.

3. Under this condition, the balance of the two voltages provides a zero voltage between the taps of the current source and the generated current from the voltage source becomes zero because of the internal infinite resistance of the current source.

4. As a result, the electric power generated by the two sources is zero, and therefore, the load cannot generate Joule heating because of energy conservation.

5. However, the current from the current source (not the voltage source) is not zero; therefore, we can predict that the resistance of the load must be zero.

A summary of our theory and numerical calculations is as follows. First, the strong combination of a twoelectron pair is demonstrated. Then, given that two electrons combine extremely strongly because of the spin magnetic attractive force, analytical calculations of the center-of-mass motion of the Hamiltonian of the pair eventually result in a macroscopic wave function. From this macroscopic wave function, we derive a London equation using the concept of an internal toroid. The key point is that, when a sample exhibits a Meissner effect, it should release the additional energy from the internal magnetic field as a discharge
\end{abstract}


current, which involves a negative voltage. Based on the inductance of this toroid, an equivalent circuit is produced. Using this circuit, we simulate this phenomenon, which results in the generation of a negative voltage and evidence of the Meissner effect, in addition to zero voltages and non-zero currents for the sample.

Keywords: temperature-independent superconductivity, circuit-approached superconductivity, electron pair, Bose-Einstein condensation, large superconducting energy gap, London equation, Meissner effect, macroscopic wave function, critical current density

\section{Introduction}

This paper proposes a new method to generate temperature-independent superconductivity. This study is significant because

1. The method generates superconductivity without refrigeration;

2. Its critical current is sufficiently high;

3. It does not require specific substances or the mounting of specific setups; and

4. The mechanism of this new type of superconductivity is explained in terms of condensed matter physics.

The principle of our superconductivity is very simple. First, a voltage source, a current source, and a load are connected in series, and then the outputs of the voltage and current sources are adjusted so that the voltage of the load and the voltage of the voltage source are balanced. Under this condition, the voltage between the taps of the current source becomes zero. Moreover, because the internal resistance of the current source is infinite, the current generated from the voltage source is zero. Considering these facts, the electric power from the two sources is zero and the resulting Joule heating from the load must be zero because of energy conservation. This implies that we can predict that the resistance of the load will become zero.

Let us consider a brief history of superconductor studies as the background for this paper. Since the initial discovery of superconductivity, more than a century has passed. During this time, many significant achievements have been made. For example, Bardeen-Cooper-Schrieffer theory [4], which found that the carrier of a superconductor has the combination of a Cooper pair, was established. Then, high-temperature superconductors whose critical temperatures are beyond that of $\mathrm{LN}_{2}$ were found. Further, $\mathrm{MgB}_{2}$ [5-6] and Fe-based superconductors [7-8] appeared. These superconductors promoted studies in condensed matter physics [9-13]. However, the common weak point of the above superconductors is that they require refrigeration. 
Approximately 8 years ago, our group demonstrated a new type of superconductivity using theoretical and experimental techniques [1-3]. This superconductivity is generated when a diffusion current from a current source is supplied to a doped semiconductor and an electrostatic field from a condenser cancels the Ohmic voltage of the semiconductor. As a result, the internal voltages are zero but the current remains. Accordingly, this new superconductivity bypasses the problem of refrigeration because it is not related to temperature.

In this paper, we describe another new type of superconductor, which demonstrates extreme progress compared with the above-mentioned superconductivity discovered 8 years ago. To distinguish these two new types of superconductivity, we will refer to the one developed 8 years ago as PNS (referring to the Previous New Superconductivity).

Compared with PNS, the present superconductivity defers in the following aspects.

1. The critical current density is much larger. This fact is important when considering practical applications. In PNS, the critical current is less than $10 \mu \mathrm{A}$, which prevents it from being used in practical applications; however, the critical current of the present superconductivity is estimated to be $2.8 \times 10^{10} \mathrm{~A}$. This value will pose no problems for practical applications.

2. It is clear that the mechanism is the Meissner effect. In PNS, we knew that the superconductor discharged a current as a result of the application of static magnetic fields. However, the details of that mechanism were not clear. In the present study, we were able to derive the concrete London equation and identify the mechanism as the Meissner effect. A key point is that the sample in an experiment discharges a current to release the additional energy derived from the magnetic field energy.

3. Numerical simulations result in clear superconductivity. In PNS, we could not establish a simulation method; however, the present superconductivity system implies a pure electrical circuit, which enabled us to employ simulation software for electrical circuit calculations (e.g., the PSIM software) by introducing an equivalent circuit. The use of this simulation method enabled us to investigate this system via various approaches.

To conclude, the mechanism of our new superconductivity including PNS enables a comprehensive understanding of the generated superconductivity.

The mechanisms for pairing an electron pair and undergoing Bose-Einstein condensation are the same here as in PNS; however, we will provide a review of the outline of our theory. First, the balance of two voltages and a diffusion current result in a specific electron concentration distribution. Using this distribution and the Poisson equation, a new electric field and a transient attractive potential are generated. Because of this solution of the Poisson equation, the Coulomb repulsive potential as the solution of the Poisson equation does not appear at the macroscopic scale, which results in the extreme 
short-range approach of the two electrons to each other. Under the condition that the kinetic energy in terms of the relative motions dominates the lattice energy (which involves the concept of the critical current density), the two electrons combine via the spin magnetic potential. This spin magnetic potential implies a superconducting energy gap that is much larger than normal heating at room temperature. Therefore, the combination of the two electrons is not destroyed by normal heating. Given that the two electrons bind very strongly, we consider the center-of-mass motion of an electron pair. As a result, the entire wave function of the center-of-mass motion converges to a macroscopic wave function.

Using this macroscopic wave function, this paper describes the derived London equation. In this process, we introduce the concept of an internal toroid and its inductance $L$. Calculating the value of the above-mentioned inductance $L$, we introduce an equivalent circuit. Using this equivalent circuit and the PSIM software, the time dependences of the voltages and current on the sample can be found. As a result, the voltage of the sample converging to zero experiences a negative voltage immediately prior to the transition time, which is an evidence of the Meissner effect. Even though the values of the resistances and currents were varied, the result of our superconductivity with negative voltages remains.

In summary, we propose new type of circuit-approached and temperature-independent superconductivity. As a result, we obtain a very large critical current, the Meissner effect is demonstrated both analytically and numerically, and the sample resistance becomes zero.

The significance of the present superconductivity is that it can be obtained very simply without refrigeration and without preparing specific substances or setups. Such achievements have not yet been obtained in conventional superconductor studies.

In this paper, we first review the characteristics of both the voltage and current sources. Then, the principle of our system is described. The mechanism of pairing and forming the macroscopic wave function is discussed theoretically by obtaining the London equation and formulating the critical current density equation. The Methods section proposes an equivalent circuit with calculations of the inductance $L$ of the toroid. In the Results section, we calculate concrete values of the critical currents and use the PSIM software to calculate the time dependences of the voltages and currents in the sample. 


\section{Principle}

\subsection{Review of voltage and current sources}

In our proposed system, voltage and current sources are employed, which are elements of a general electrical circuit. Figure 1 shows schematics of these sources. The voltage source generates a constant voltage while its current is varied depending on the connected load. The internal resistance of this voltage source is ideally zero. Conversely, the current source supplies a constant current (not voltage) to the load, which is not related to the electrical resistance of the load. Therefore, its voltage is varied depending on the resistance of the load. In contrast to the voltage source, the internal resistance of the current source is ideally infinite.
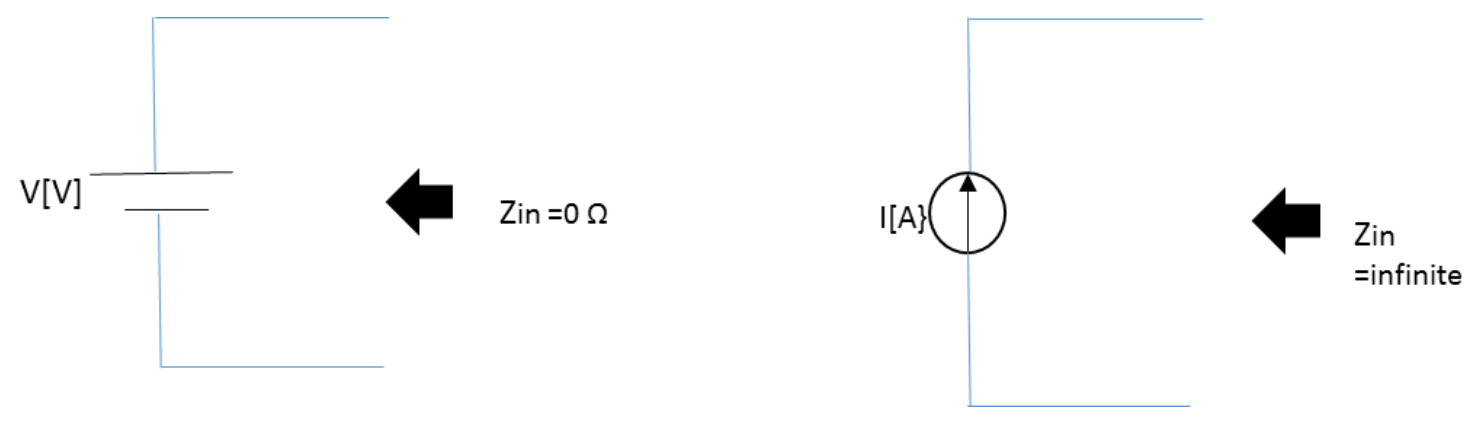

Fig. 1

Schematics of the voltage and current sources. The left panel shows the voltage source, which supplies a constant voltage regardless of the value of the connected load. Conversely, the right panel shows the current source, which provides a constant current regardless of the value of the load. This current supply is achieved via the employment of collector currents of transistors. An important point is that their internal resistances, i.e., the input impedances $Z_{i n}$, are opposite to each other. The internal resistance of the voltage source is zero, whereas the internal resistance of the current source is infinite.

\subsection{Principle}

As shown in Fig. 2, a voltage source, a current source, and a load are connected in series. As previously mentioned, the internal resistance of the voltage source is zero, whereas the internal resistance of the current source is infinite. Moreover, the output voltage is equal to the voltage from the load, as derived via Ohm's law. Considering these points, the following can be said:

1. The generating current of the voltage source is zero because of the infinite resistance of the current source. Therefore, this source generates only the voltage $V$.

2. For the current source, because of the balance of the two voltages of the voltage source and the 
load, the voltage between the taps of the current source is zero. Therefore, this source supplies only the current $I$ to the load.

These characteristics are listed in Table 1.

Considering the above, we see that neither source generates electric power. Therefore, energy conservation requires that the load not receive any energy and that the load not generate Joule heating. Because the current $I$ exists in the load because of the current source, the absence of Joule heating by the load results in zero electric resistance. Therefore, we can predict that this system, in principle, will result in a new type of superconductivity. However, before we reach this conclusion, it is necessary to theoretically examine the mechanism of the superconductivity in terms of condensed matter physics and demonstrate that the Meissner effect is generated.

Table 1 Comparisons characteristics between voltage and current sources acting our system

\begin{tabular}{l|ccc}
\hline & Its voltage & Its current & Its electric power \\
\hline Voltage source & Constant V $[\mathrm{V}]$ & $0[\mathrm{~A}]$ & $0[\mathrm{~W}]$ \\
& & & \\
Current source & $0[\mathrm{~V}]$ & Constant I $[\mathrm{A}]$ & $0[\mathrm{~W}]$ \\
\hline
\end{tabular}




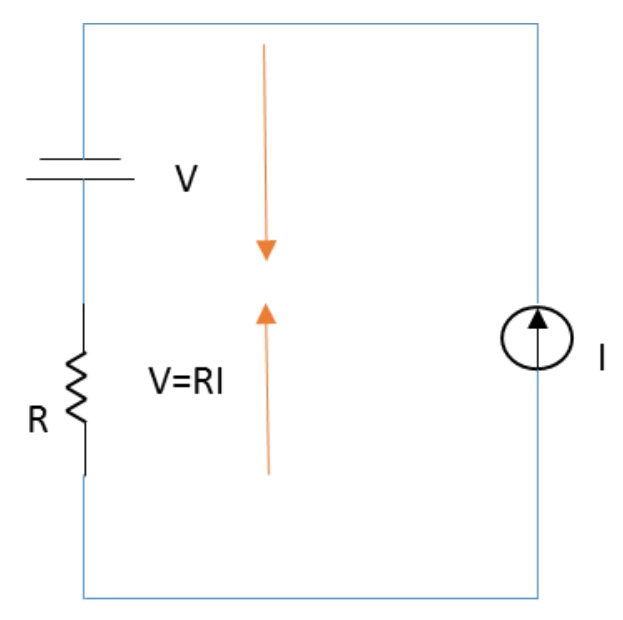

\section{Fig.2}

Schematic of the system generating the superconductivity. After setting a current $I$ from the current source, the output of the voltage source is adjusted to balance the Ohmic voltage from the load. In this way, the voltage over the current source becomes zero but the output current $I$ remains, and the current from the voltage source becomes zero because of the infinite internal resistance of the current source but the output voltage is kept constant. Therefore, the electric power from the two sources is zero, which implies that the load does not generate Joule heating because of energy conservation. Therefore, we can predict that the resistance of the load will become zero.

\section{Theory}

\subsection{Spatial electron concentration at transient state}

First, we assume that the voltage of the voltage source and the voltage of the load are equal. Then, the diffusion current of the current source is introduced. Considering the conductivity, introducing an average electric field, and substituting a diffusion constant result in a specific and special electron concentration. The voltage balance is

$$
\begin{aligned}
& V_{E}=V=R I, \\
& R=\rho \frac{l}{s},
\end{aligned}
$$

where $V_{E}, V, R$, and $I$ denote the voltage from the voltage source, the voltage of the load, the resistance of the load, and the current from the current source, respectively. In Eq. (1-2), $\rho, S$, and $l$ denote the resistivity, the area of the load, and the length of the load, respectively. The diffusion current $I$ is expressed as 


$$
I=S q D \frac{d n}{d \xi}
$$

where $q, D$, and $n$ denote the electron charge, the diffusion constant, and the electron concentration, respectively. Therefore, the Ohmic voltage becomes

$$
V=\rho \frac{l}{S} S q D \frac{d n}{d \xi}=\frac{l}{\sigma} q D \frac{d n}{d \xi}
$$

where $\sigma$ denotes the conductivity of the load. Here the following equations are substituted:

$$
\begin{gathered}
\sigma=q n \mu, \\
D=\frac{\mu k_{B} T}{q}, \\
V=E_{i} l,
\end{gathered}
$$

where $\mu, k_{B}, T$, and $E_{i}$ denote the average mobility, the Boltzmann constant, the temperature, and the average local electric field, respectively. Therefore, the local electric field is

$$
E_{i}=\frac{k_{B} T}{q n} \frac{d n}{d \xi}
$$

Solving this equation gives

$$
n=n_{0} \exp \left(\frac{q E_{i}}{k_{B} T} \xi\right)
$$

\subsection{Transient state attractive potential and coiled new electric field [1-3]}

We employed the Poisson equation to derive the interaction potential between two electrons. Equation (6) gives the concentration in terms of the electrons; substituting this concentration into the Poisson equation produces the following interaction potential:

$$
V_{\alpha}=-\frac{n_{0} k_{B}^{2} T^{2}}{q \varepsilon E_{i}^{2}} \exp \left(\frac{q E_{i}}{k_{B} T} \xi\right)
$$

where $\xi$ is the relative macroscopic distance between the two electrons. Hereafter, we refer to this potential as the transient state potential.

In the process of solving the Poisson equation, we derived the following new electric field:

$$
E_{m}=-\frac{n_{o} k_{B} T}{\varepsilon E_{i}} \exp \left(\frac{q E_{i}}{k_{B} T} \xi\right)
$$

The above electric field has features of both an electrostatic field and a current. It is necessary to define the circle integral of $E_{m}(\xi)$ (i.e., the voltage) because we need to ensure that $E_{m}(\xi)$ is not divergent. Note that the circle integral is only undefined at $\xi=0$ because the normal ordinate of the relative coordinate must be an edge of the integral and this normal ordinate should not be part of the path of 
the integral. This exception allows $E_{m}$ to have both a static component and a component related to the current. That is, this new electric field forms a "coiled electric field." The spatial integral of Eq. (8) results in a negative voltage. As discussed later, we will see that, immediately prior to the transition from the normal state to the superconducting state, this negative voltage appears in our simulation. It is a very important to note that the Coulomb repulsive interaction is also determined using Poisson's equation. Accordingly, this repulsive interaction does not appear at a macroscopic scale instead of $V_{\alpha}$.

\subsection{Combination of two electrons and critical current density}

The transient state attractive interaction potential $V_{\alpha}$ results in two electrons that approach each other until their distance is only the lattice diameter due to the lack of the Coulomb repulsive interaction at the macroscopic scale. The above-mentioned potential $V_{\alpha}$ functions at the macroscopic scale, and therefore, the transient state up to the combination of the two electrons is a macroscopic phenomenon. Only when the potential $V_{\alpha}$ vanishes do we need to consider the microscopic scale. As described later, however, at this moment, when the potential $V_{\alpha}$ vanishes, the relative kinetic energy also vanishes. Therefore, we do not need to consider the Hamiltonian of the quantum mechanics at this stage.

As a result of the transient interaction $V_{\alpha}$, the two electrons are located at a lattice, and at a lattice (i.e., macroscopic variable $\xi=0$ ), the total energy $E_{T}$ of the system is expressed as follows:

$$
\begin{aligned}
E_{T} & =V_{\alpha}(\xi=0)+V+V_{p}, \text { and } \\
V_{\alpha}(\xi & =0)+P=0 .
\end{aligned}
$$

Here, $V$ is the spin magnetic potential [1] expressed as

$$
V=-\frac{q^{2} \hbar^{2}}{16 \pi m^{2}\left|z_{m}\right|^{3}}
$$

where $m$ and $z_{m}$ denote the electron mass and the microscopic relative coordinate, respectively. In Eq. (9), $P$ and $V_{p}$ are the kinetic and zero-point energies in terms of the Debye temperature at the lattices, respectively. $V_{p}$ is expressed as follows:

$$
V_{p}=\frac{1}{2} \hbar \omega_{D}=\frac{1}{2} k_{B} \theta_{D}
$$

where $\omega_{D}$ and $\theta_{D}$ indicate the Debye angular frequency and temperature, respectively. The following conditions should be satisfied for the two-electron combination:

$$
\begin{aligned}
& \quad\left|V_{\alpha}(\xi=0)\right| \geq V_{p}, \\
& |V| \geq P .
\end{aligned}
$$

For Eq. (12-1) to be satisfied, both electrons must be located at the zero point of a lattice. This condition, i.e., critical current density, will be considered later. To satisfy Eq. (12-2), the microscopic relative distance $z_{m}$ (i.e., the coherence) should be 


$$
z_{m} \leq 1.0 \times 10^{-9} \mathrm{~m}
$$

If Eq. (12-1) is satisfied, then, Eqs. (12-2) and (13) are also satisfied because the zero-point energy implies the quantum fluctuation energy of a lattice. Therefore, only the spin magnetic potential $V$ remains, and the entire energy takes on a negative value, which implies that the two electrons have a net combination, in which a collision between the two electrons becomes completely inelastic. Consequently, these two electrons combine to form a pair.

Even though the kinetic energy in terms of the relative motions exists in the transient state, as mentioned, in the steady state, only the spin attractive force $V$ remains:

$V=-\frac{q^{2} \hbar^{2}}{16 \pi m^{2} a^{3}}$

where $a$ denotes the constant coherence of an electron pair.

Therefore, this potential is taken to be the superconducting energy gap of our superconductivity. As mentioned, the two-electron pair combines at the distance of a lattice, which is typically estimated to be $1 \AA$, and, therefore, the magnitude of the energy gap is estimated to be approximately $10^{-18} \mathrm{~J}$. The typical room temperature energy $k_{B} T$ (where the temperature $T=300 \mathrm{~K}$ ) is approximately on the order of $10^{-21} \mathrm{~J}$. Therefore, this superconducting energy gap is much larger than that of room temperature. This implies that the individual electron pair will not be destroyed via normal heating.

At the critical point, i.e., in the case where the inequality of Eq. (12-1) is maximum,

$$
\frac{n_{0} k_{B}^{2} T^{2}}{\varepsilon E_{i}^{2}}=\frac{1}{2} k_{B} \theta_{D}
$$

From this equation, the concentration $n_{0}$ can be expressed as

$$
n_{0}=\frac{\varepsilon E_{i}^{2}}{k_{B} T^{2}} \frac{1}{2} \theta_{D}
$$

In Eq. (14), considering the second equation of Eq.(9), the left-hand side of Eq. (14) implies a kinetic energy $P$. Therefore,

$$
\frac{n_{0} k_{B}^{2} T^{2}}{\varepsilon E_{i}^{2}}=\frac{1}{2} m v^{2},
$$

where $m$ and $v$ denote the mass of the electron and the velocity in terms of the relative motions, respectively. Substituting Eq. (15),

$$
\frac{\varepsilon E_{i}^{2}}{k_{B} T^{2}} \frac{1}{2} \theta_{D} \frac{k_{B}^{2} T^{2}}{\varepsilon E_{i}^{2}}=\frac{1}{2} m v^{2} .
$$

That is,

$$
\frac{1}{2} k_{B} \theta_{D}=\frac{1}{2} m v^{2}
$$


Therefore, the relative velocity is derived to be

$$
v=\sqrt{\frac{k_{B} \theta_{D}}{m}}
$$

Consequently, the critical current density equation is obtained such that

$$
j_{c}=q n_{0} v=q \frac{\varepsilon E_{i}^{2}}{k_{B} T^{2}} \frac{1}{2} \theta_{D} \sqrt{\frac{k_{B} \theta_{D}}{m}} .
$$

Note that, in the Results section, we will calculate numerical values of the critical current density using Eq. (20).

\subsection{Macroscopic wave function and the London equation}

Given that a two-electron pair binds strongly, from the discussion in the previous sections, we now precede to a discussion of Bose-Einstein condensation and the macroscopic wave function.

The Hamiltonian equation of an electron pair in terms of the center-of-mass motion is expressed as

$$
H=-\frac{\hbar^{2}}{2(2 m)} \frac{d^{2}}{d x^{2}}+U
$$

The potential $U$ in the above equation indirectly implies the electrostatic potential of the two electrons, i.e.,., the external fields:

$$
U=-\int_{x_{1}}^{x_{2}} 2 q E_{i} d x=-2 q E_{i}\left(x_{2}-x_{1}\right)
$$

where $x_{1}$ and $x_{2}$ denote the positions of the two electrons.

The local placements at the identical lattice of the two electrons (i.e., the local and strong combination of the two electrons at a lattice) imply that $x_{1}=x_{2}$. Employing the Hamiltonian equation, a wave function of the pair in terms of the center-of-mass motion can be obtained. Note that, at the moment when the electrons have an identical location at a lattice, the potential $U$ in the Hamiltonian equation converts to an eigenvalue (i.e., the kinetic energy in terms of the center-of-mass motion) that stems from the local electric field $E_{i}$. Accordingly, the distribution of the local electric field $E_{i}$ within the lattice vanishes.

$$
\varphi_{i}=\gamma \exp \left[\frac{2 m \mu_{i} E_{i}}{\hbar} j x\right]
$$

where $\mu$ denotes the mobility of the pair at the lattice. Using the following equations,

$$
\begin{aligned}
& \frac{J}{\sigma_{i}}=E_{I} \text { and } \\
& \sigma_{i}=\frac{2 q}{\Delta V_{i} / i} \mu_{i},
\end{aligned}
$$


the wave function of an electron pair can be derived such that

$$
\varphi_{i}=\gamma \exp \left[\frac{2 m j}{\hbar} x J \frac{\Delta V_{i} / i}{2 q}\right],
$$

where $\sigma_{\mathrm{i}}, \Delta V_{i}$, and $J$ denote the conductivity at the lattice, the variation quantity of the volume with respect to the increase in the index $i$, and the current density, respectively.

Equation (24-1) implies the proportion of Joule electric and electrostatic fields at the lattice-scale level. The above wave function of an electron pair is indexed by $i$, and a wave function at a subsequent lattice does not interact with this wave function because the distance between the two neighboring lattices is much larger than the coherence of the two electrons. Note that, in the previous section, we mentioned that the coherence of a combined pair is estimated to be approximately $1 \AA$ (i.e.,., the typical diameter of a lattice). The entire wave function is represented in the following equation:

$$
\psi=\prod \varphi_{i}=\gamma^{i} \exp \left[\frac{2 m j}{\hbar} x \sum J \frac{\frac{\Delta v_{i}}{i}}{2 q}\right] .
$$

Assuming that the index $i$ is infinite and that the sample volume of a load is kept constant, the lattice volume $\Delta V_{i} / i$ converges to the differential $d V$. Accordingly, the countable lattice concept becomes ineffective, and a macroscopic continuous body appears. That is, we obtain a macroscopic wave function that is mandatory when considering the mechanism of the new superconductivity, and this macroscopic wave function is needed to derive a London equation (i.e., a Meissner effect):

$$
\rightarrow \eta \exp \left[\frac{2 m j}{\hbar} x \int \frac{J}{2 q} d V\right]=\eta \exp \left[\frac{2 m j}{\hbar} x a \int \frac{J}{2 q} d S\right]=\eta \exp \left[\frac{m I}{\hbar q} a j x\right] .
$$

In Eq. (27), the wave number $K$ is

$$
K=\frac{m I}{\hbar q} a=\frac{I}{2 M} a,
$$

where $M$ denotes the spin magnetic moment of an electron and $a$ is the constant coherence in terms of our new superconductivity. The spin magnetic moment in this equation can be correlated to the combination energy (i.e., the spin interaction) from Eq. (10) when the microscopic ordinate $z_{m}$ is replaced with the constant coherence $a$.

$$
K=\frac{I}{2 \sqrt{-4 \pi a V}} V \leq 0,
$$

where $V$ denotes the spin interaction given in Eq. (10-2).

In Eq. (28-2), the macroscopic current $I$ is independent of the collision time and is therefore a superconducting current. Further, the wave number $K$ in this equation is uniform and is not related to 
space, e.g., it is not related to the lattice index $i$. Therefore, all the quantum states converge to a single state, and Bose-Einstein condensation is derived.

Let us consider the Meissner effect derived from the macroscopic wave function. As mentioned, the macroscopic wave function is

$\psi=\eta \exp \left(\frac{m I a}{\hbar q} j \mathrm{x}\right)$

In this equation, the macroscopic current $I$ appears. In general, a current needs to be continuous in space and time. In this case, therefore, the current $I$ is continuous for the output current $I$ from the current source. Accordingly, this current must generate a self-magnetic field in the load sample and, if a new superconductivity is generated, this self-magnetic field must be canceled out.

Considering the above self-magnetic field $A$, the Aharonov-Bohm effect [14] can be introduced to the macroscopic wave function, Eq. (27):

$$
\psi_{A}=|\psi| \exp \left[\left(\frac{m I a}{\hbar q} x+\frac{q}{\hbar} \int A d s\right) j\right] .
$$

Based on the property of phases,

$$
\frac{m I a}{\hbar q} x+\frac{q}{\hbar} \int A d s=2 n \pi
$$

Assuming that the integer $n=0$,

$$
\frac{m I a}{\hbar q} \mathrm{x}=-\frac{q}{\hbar} \int A d s .
$$

The differentials are then applied to both sides:

$$
\frac{m I a}{\hbar q}=-\frac{q}{\hbar} A d s
$$

That is,

$$
\frac{m a}{\hbar q} \int j_{n} d S=-\frac{q}{\hbar} A d s
$$

Assuming a differential for integrals for the surface and the line equal to the coherence $a$, which is very small, Eq. (33) becomes

$$
\frac{m a}{\hbar q} j_{n} \times a^{2}=-\frac{q}{\hbar} A \times a .
$$

Therefore, we derive a London equation:

$$
j_{n}=-\frac{q^{2}}{m a^{2}} A .
$$




\subsection{Shield current distribution and the interpretation of derived London equation}

When considering a sample with a cylindrical shape, it is necessary to introduce a toroid whose large radius corresponds to that of the cylindrical sample. Then, we consider the renewable coordinate that is tangential to the large circumference of the toroid as corresponding to the $z$-axis in cylindrical coordinates; see Fig. 3. We refer to this type of coordinates as the "specific cylindrical coordinates."

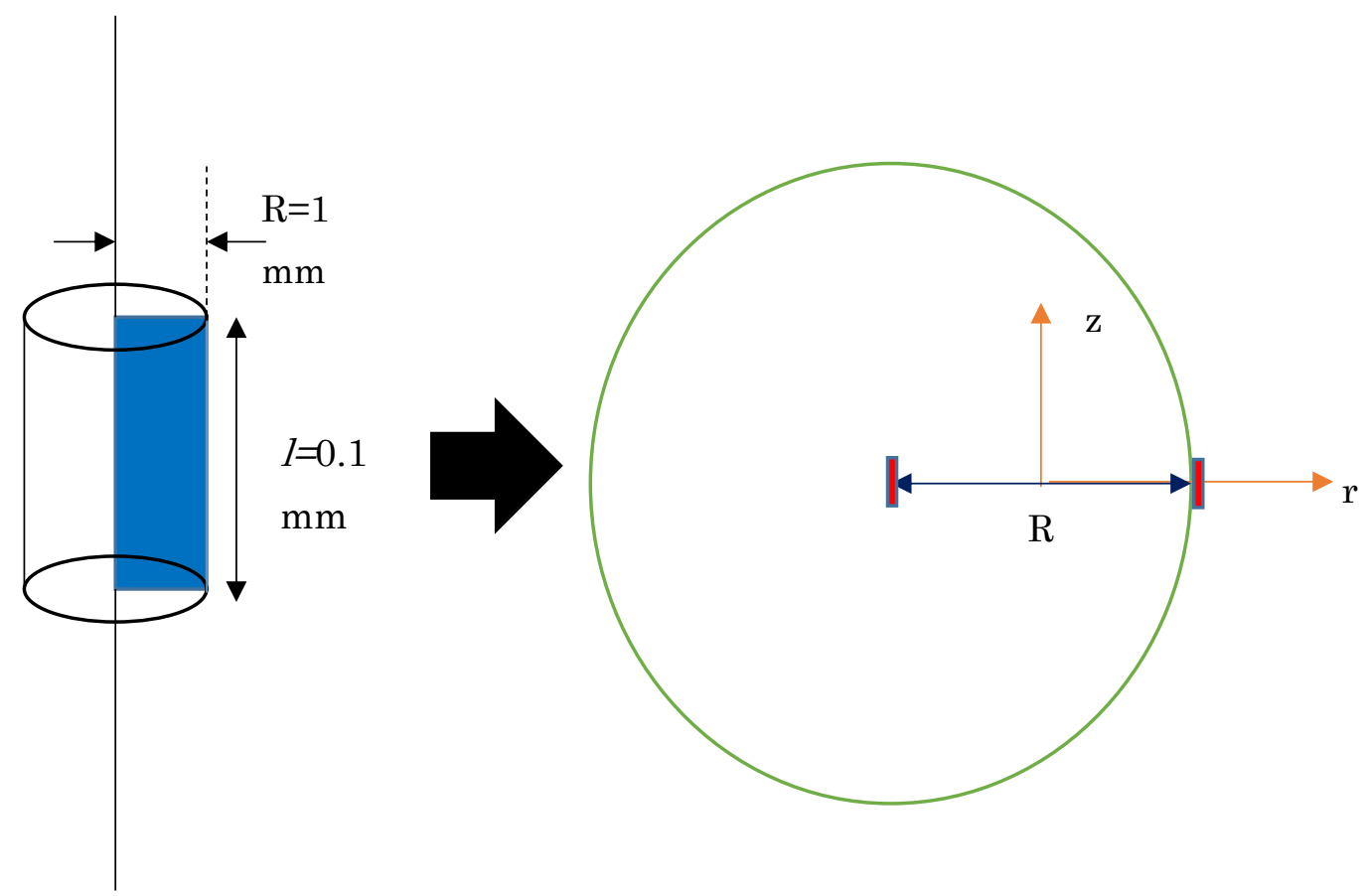

Fig.3

Schematic of the specific coordinates of a toroid. The left panel indicates a load whose macroscopic shape is cylindrical, and $R$ is a radius that is identical to the large radius of a toroid. The right panel shows a cross section of an elemental coil of the toroid. The red square is a cross section of the elemental coil, which is located in the $z-r$-axes of the specific cylindrical coordinates. Note that this is a schematic and therefore an elemental coil is actually very small because its height along the $z$-axis corresponds to the coherence $a$ of our superconductivity (it is a so-called two-dimensional elemental coil) and that there is generally a $\phi$-axis in cylindrical coordinates but the actual area that the magnetic field vectors cross perpendicularly depends on $l$, depicted as the blue area in the left panel.

From the initial time to the transition time $t_{c}$, there is a normal self-magnetic field distribution $B_{0}$ in the sample along the direction of the $z$-axis of the specific cylindrical coordinates. Because of the 
formation of the macroscopic wave function at the time $t_{c}$, however, $B_{0}$ is canceled by a magnetic field whose direction is in the $z$-axis of the specific cylindrical coordinates generated by the current from Eq. (35). Therefore, the internal net magnetic flux density becomes zero despite the existence of the current $I$. However, it is important to note that a large toroid inductance $L$ still exists because the inductance is generally calculated only using the geometrical parameters of a coil. Note that, from energy conservation, the magnetic field energy derived from $B_{0}$ is transformed as a discharged current, which generates a temporal negative voltage, as mentioned previously.

\section{Method}

\subsection{Equivalent circuit}

In the previous section, it was found that a toroid inductance $L$ is produced in the sample. Considering this, we can introduce an equivalent circuit for our system, shown in Fig. 4, which has an inductance $L$. That is, the sample has both a resistance $R$ and an inductance $L$. It is not necessary to worry that, at the initial time $(t=0)$, the inductor might influence the balance of the two voltages because, at that time, the impedance of the inductor is infinite. Therefore, at the initial time $(t=0)$, the equivalent circuit is identical to that shown in Fig. 2 (i.e., our proposed system).

For the numerical calculations, we employed the PSIM electrical circuit software, which can be purchased from their website. 


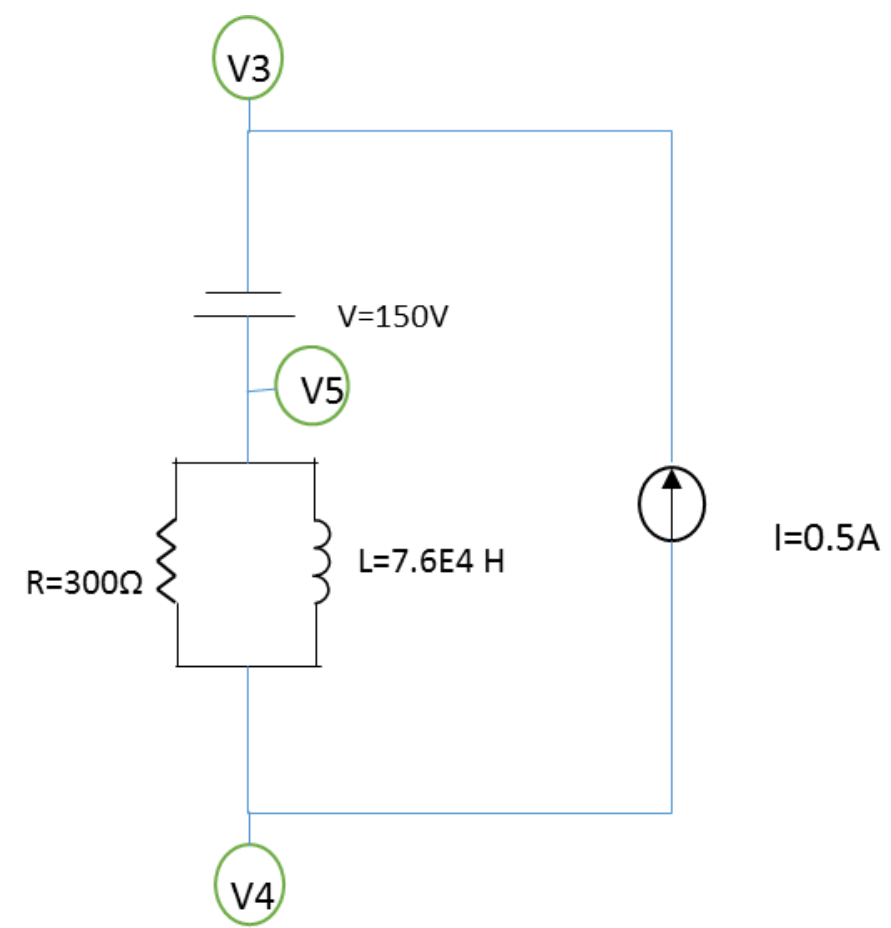

Fig.4

The circuit used for the numerical calculations. Note that V3, V4, and V5 indicate the probes of the electrostatic potentials and that the parallel $R$ and $L$ imply the equivalent circuit for our sample. If the value of $R$ is varied, then the outputs of the voltage source are also varied to maintain the voltage balance.

\subsection{Calculation of inductance $L$}

To simulate the equivalent circuit, we need to estimate a concrete value of the inductance $L$, which is determined by the geometrical factors of the load. We show an example shape of the load in Fig. 3. As mentioned, it is necessary to consider the specific cylindrical coordinates, i.e.,., a toroid. The inductance of a toroid is given as

$$
L=\frac{\mu_{0} N^{2} S}{2 \pi R},
$$

where $\mu_{0}, N, S$, and $R$ denote the magnetic permeability in a vacuum, the turn number, the area, and the radius of the toroid, respectively. Note that the area $S$ is indicated in Fig. 3 as a blue surface. In this equation, the turn number is derived from the fact that each element coil of the toroid is distributed with a relative distance that is equal to the coherence $a$ of our superconductivity:

$$
N \approx \frac{2 \pi R}{a}
$$

where the coherence $a$ is approximated as $1 \AA$. From Fig.3, the inductance L is calculated as

$L \approx 7.6 \times 10^{4} \mathrm{H}$. 
This value appears to be very large; however, as will be discussed later, unless the inductance of a toroid is sufficiently large, we cannot obtain a sufficiently large critical magnetic field. This is important when considering the load to be a normal coil that is chosen to generate large magnetic fields by changing the superconductivity.

\subsection{Resistance of a lord}

In general, the value of the resistance of a load is determined by both the conductivity and its shape. In this paper, under the condition that the shape of the load of the sample is kept constant, the conductivity is varied because the sample is considered to be a doped semiconductor. That is, while the inductance $L$ is kept constant, the resistance of the sample is varied.

\section{Results and Discussion}

\subsection{Circuit simulations}

First, we show the result when the load is $300 \Omega$ and the input current is $0.5 \mathrm{~A}$. Figures $5 \mathrm{a}-5 \mathrm{c}$ indicate the electrical potentials according to Fig. 4. Figure 6 shows the time dependence of the current that exists in the sample.

At the initial time, the difference between V3 and V4 is nearly zero, which implies the tap voltage of the current source. However, at $t=1000 \mathrm{~s}$, V5 becomes nearly zero and V4 becomes zero before taking on negative values. In addition, the current in Fig. 6 reaches a constant. This implies that the tap voltage between V5 and V4, i.e., the voltage of a sample, becomes zero but the current is retained. As mentioned, a negative voltage occurs immediately prior to the time of the transition. The negative value at V4 implies a demonstration of the Meissner effect. However, this negative value may be noise; therefore, we measured the electrical potentials after changing the value of the resistance of the load to $100 \Omega$. 


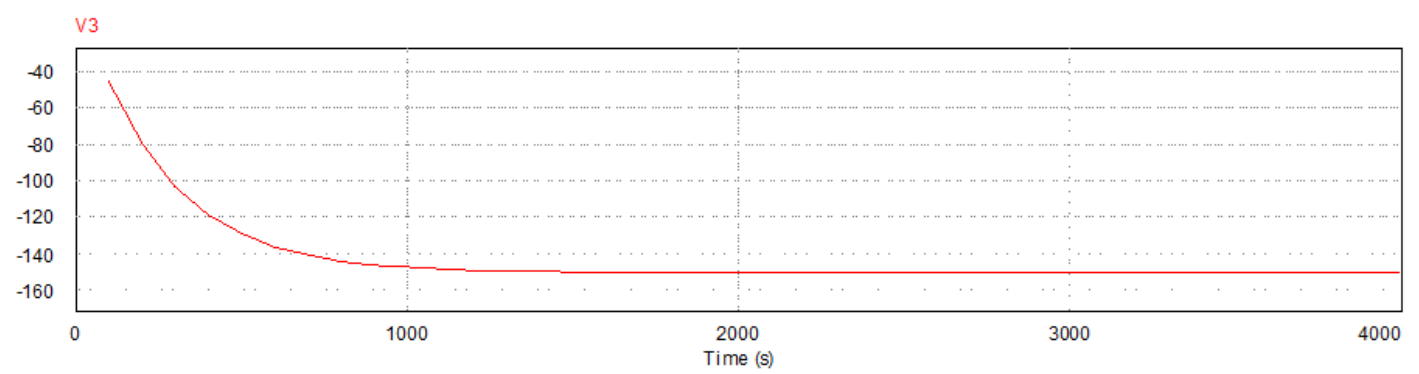

[a]

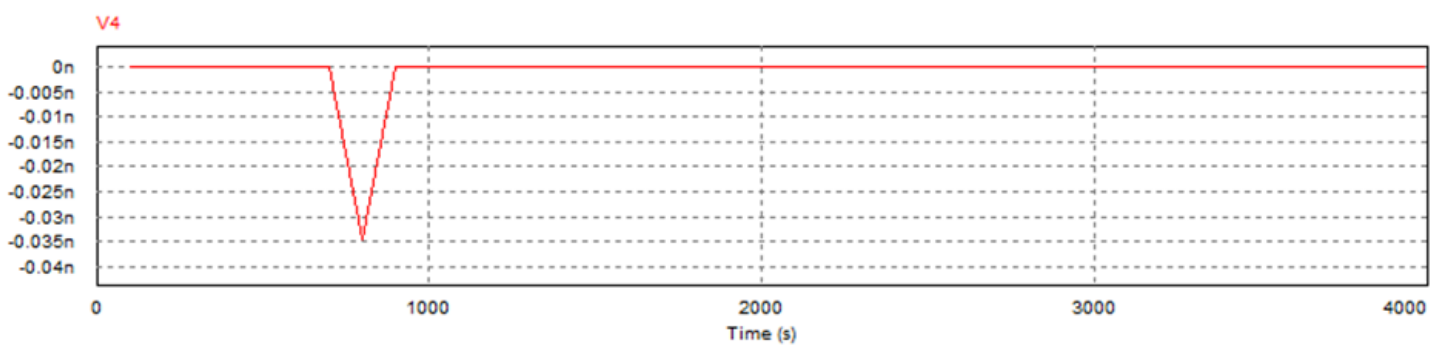

[b]

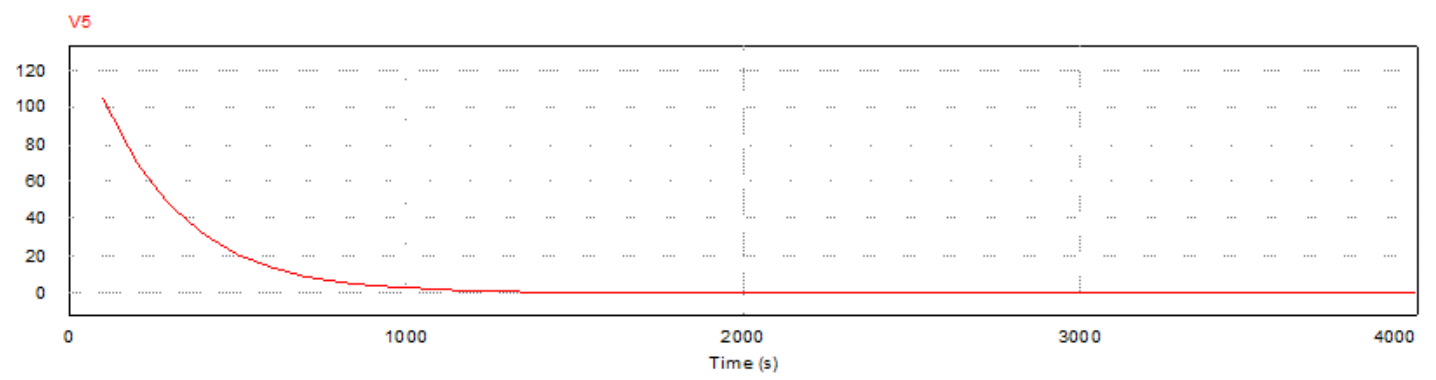

[c]

Fig.5

Time dependences of the electrical potentials. Note that the perpendicular axes indicate the electrostatic potential [V]. The probe names correspond to those in the schematic circuit shown in Fig. 4. V5 converges to nearly zero after $1000 \mathrm{~s}$, whereas V4 indicates negative voltages prior to that time. This negative voltage implies that the additional energy derived from the self-magnetic field was released as a discharge current from the inductance of the toroid. 


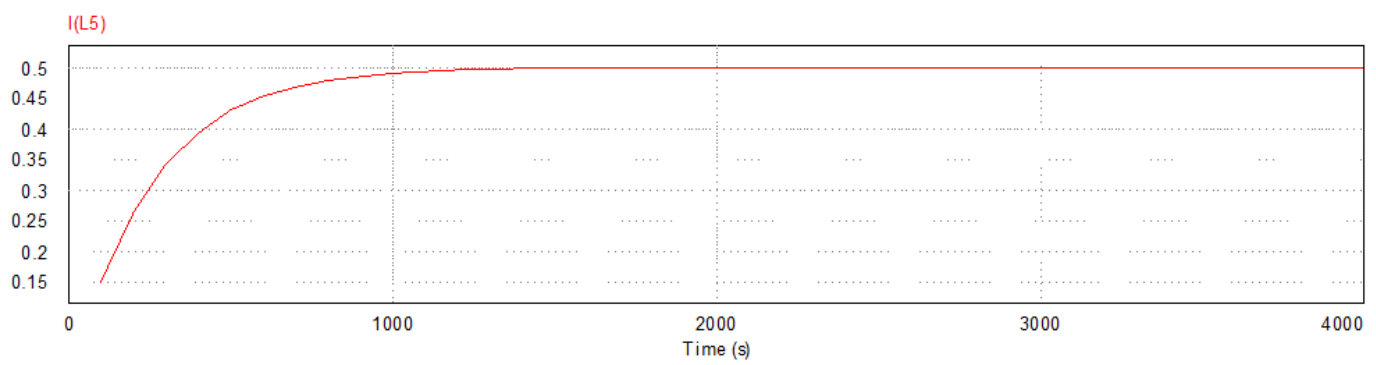

\section{Fig.6}

Time dependence of the current in the sample. Note that the perpendicular axis indicates the current [A]. Corresponding to the behaviors of the electric potentials, the current converges to a steady state after $1000 \mathrm{~s}$.

In Figs. 7a-7c, each electrical potential in the case with $100 \Omega$ is presented, whereas Fig. 8 shows the characteristics of the current in the sample. The behaviors of each electrical potential and the current are the same as in the case with $300 \Omega$; however, the transition time becomes longer shifting to $2000 \mathrm{~s}$. As an important result, V4 again exhibits a negative value similar to that in the case with $300 \Omega$. Therefore, we can conclude that negative voltages appear immediately prior to the transition time demonstrating the Meissner effect predicted by our theory.

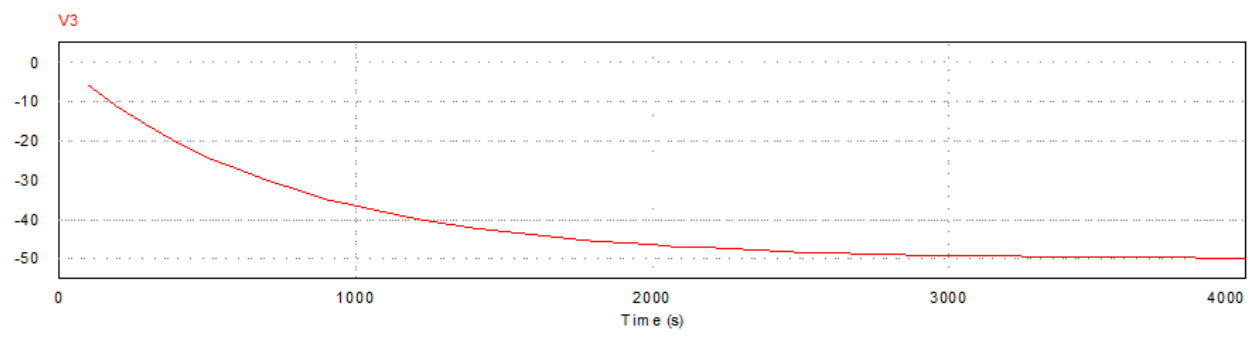

[a]

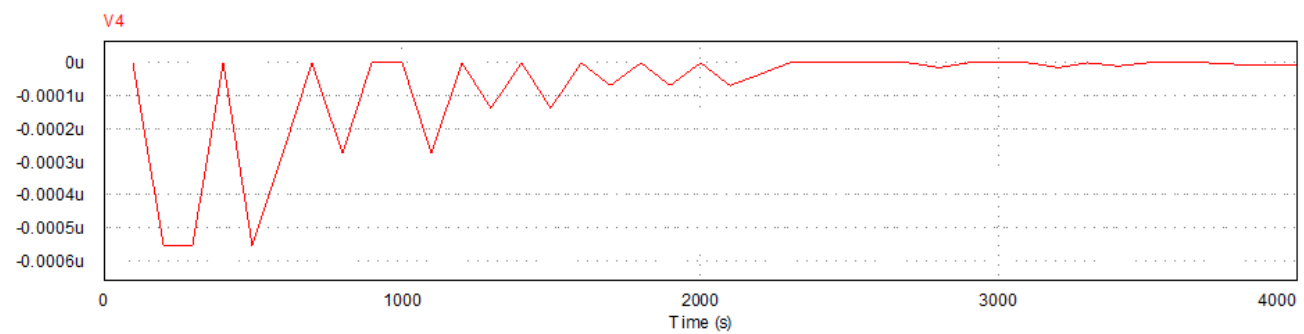

[b] 


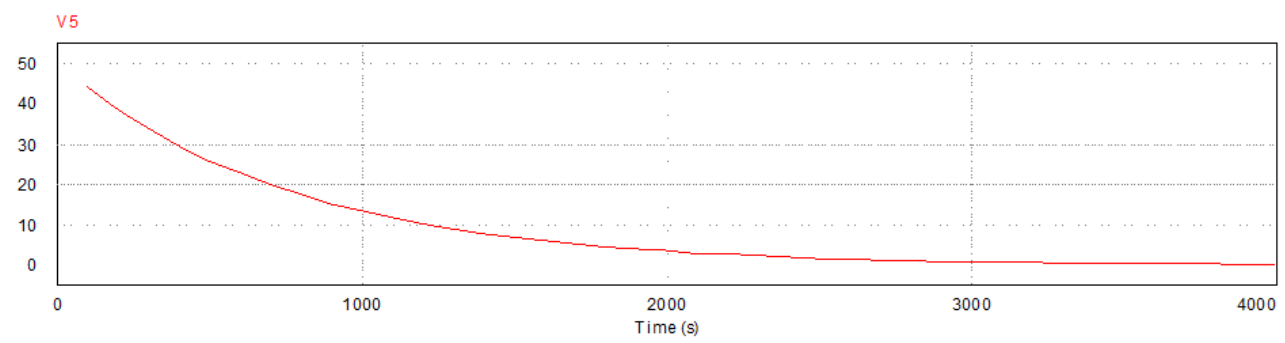

[c]

Fig.7

Time dependences of each electrical potential in the case where the load is $100 \Omega$. Note that the input current is kept constant at $0.5 \mathrm{~A}$ but that the input voltage of the voltage source was changed to $50 \mathrm{~V}$ to balance the voltage. The V5 probe shows that, after $2000 \mathrm{~s}$, the electrical potential converges to zero, whereas the V4 probe indicates negative voltages prior to that time. Therefore, we can see that the surplus energy from the self-magnetic field was released and that this occurred immediately prior to the transition time.

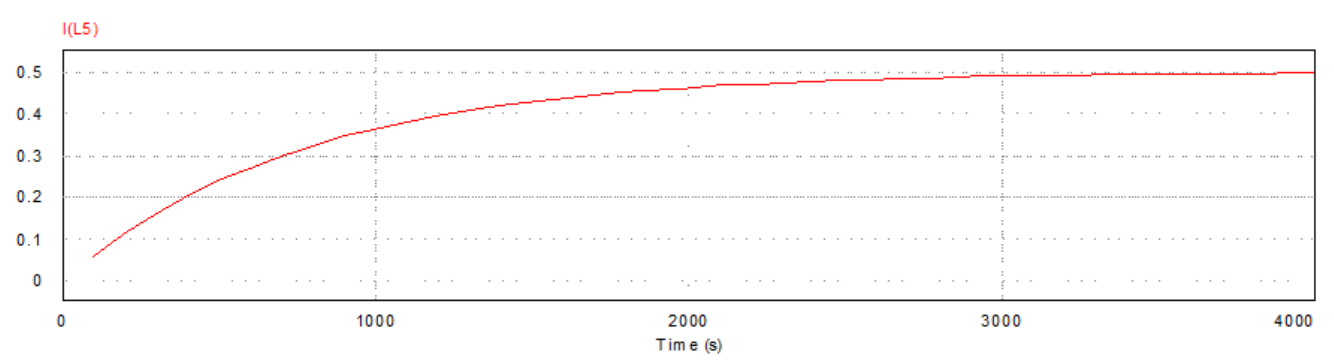

\section{Fig.8}

Time dependence of the current in the case where the load is $100 \Omega$. As shown, the current converges to $0.5 \mathrm{~A}$ at the time corresponding to the convergence of the electric potentials.

\subsection{Values of critical current and comparison with that in PNS}

Using Eq. (20), we calculated the critical current. When the voltage $V=100 \mathrm{~V}$ and the length of the sample is $0.1 \mathrm{~mm}$, then the average electric field is

$$
E_{i}=\frac{V_{E}}{l} \approx 1.0 \times 10^{6} \mathrm{~V} / \mathrm{m},
$$

where $V_{E}$ and $l$ denote the voltage from the voltage source and the length of the load, respectively. Considering that the macroscopic radius of the load is approximated as $1 \mathrm{~mm}$, the critical current $I_{c}$ is calculated such that 


$$
I_{c}=2.8 \times 10^{10} \mathrm{~A} .
$$

Table 2 lists the physical constants used in these calculations.

Table 2 Physical constants in the calculation of jc

Temperature $\mathrm{T}$

Electron charge $\mathrm{q}$

Boltzmann constant $\mathrm{k}_{\mathrm{B}}$

Debye temperature $\theta_{\mathrm{D}}$

Permittivity $\varepsilon$

Length of a sample 1

Output voltage of a voltage source $\mathrm{V}_{\mathrm{E}}$
$300 \mathrm{~K}$

$1.6 \times 10^{-19} \mathrm{C}$

$1.38 \times 10^{-23} \mathrm{~J} / \mathrm{K}$

$120 \mathrm{~K}$

$8.8 \times 10^{-12} \mathrm{~F} / \mathrm{m}$

$10^{-4} \mathrm{~m}$

$100 \mathrm{~V}$

Table 3 Additional physical constants in calculating jc in PNS

Capacitance $\mathrm{C}$

Applied voltage to condenser $\mathrm{V}_{\mathrm{c}}$

Permittivity $\varepsilon$

Surface area of a pole plate of condenser $\mathrm{S}_{0}$

Sored charge of condenser $\mathrm{Q}$

Surface charge density on the pole plates $\sigma$

\section{$1.0 \times 10^{-14} \mathrm{~F}$}

$1 \mathrm{~V}$

$8.8 \times 10^{-12} \mathrm{~F} / \mathrm{m}$

$0.01 \mathrm{~m}^{2}$

$1.0 \times 10^{-14} \mathrm{C}$

$1.0 \times 10^{-12} \mathrm{C} / \mathrm{m}^{2}$

The value of Eq. (40) is sufficiently large; therefore, we need not to worry about the basic limitation of the transport current.

PNS [1-3] has the same equation, Eq. (20), for the critical current density. In this case, however, the average electric fields do not stem from the voltage from the output of a voltage source but from the electrostatic fields generated by a condenser. Therefore, $E_{i}$ in Eq. (20) is derived from the surface charge density $\sigma$, which arises from the charge $Q$ on the condenser pole plates. However, because of the extremely small capacitance $C$ (approximately $10^{-14} \mathrm{~F}$ ), even when a relatively large voltage is applied to the condenser pole plates, the charge $Q$ is extremely small; in addition, the area of the pole plates is large. Therefore, the surface charge density $\sigma$ becomes very small. Considering our previous setup [1-3],

$$
E_{i}=\frac{\sigma}{\varepsilon} \approx 0.11 \mathrm{~V} / \mathrm{m} .
$$

Therefore, the critical current in this case is 


$$
I_{c, P N S}=2.8 \times 10^{-5} \mathrm{~A} .
$$

In Table 3, we indicate the additional constants used to calculate the above value.

Comparing the two critical currents, it is clear that the case proposed in this paper has sufficient technical merit. This is important when considering superconductivity applications.

\subsection{Critical magnetic field and reason why the internal toroid inductance is so large}

Let us consider the critical magnetic field in our superconductivity. In this superconductivity, even though a current exists, the net internal magnetic fields are zero in the steady state. Therefore, when an external magnetic field is applied, this surplus magnetic field energy is transformed into a discharge current. When an external magnetic field $B_{e x}$ is applied, the following equation must hold:

$$
\frac{1}{2} L I^{2} \geq \int \frac{B_{e x}^{2}}{2 \mu_{0}} d v .
$$

If the applied magnetic field $B_{e x}$ is uniform within the sample, the critical magnetic field $B_{c}$ is estimated to be

$$
B_{c}=1.7 \times 10^{5} \mathrm{~T} .
$$

If this inequality is broken, the superconductivity will vanish. This value is sufficiently large; therefore, it is possible to make a large normal coil with resistance superconductive.

\subsection{Summary of significances of this study}

Let us summarize significance of our proposed superconductivity.

\section{We need not prepare specific substances or setups.}

It is not necessary to prepare specific substances or compounds because, if the system is implemented correctly, the load is rendered superconductive. Moreover, in our previous system, we needed to prepare a setup, in which a condenser, semiconductor disk, and current leads were included. In particular, attaching the current leads to the semiconductor disk was difficult. In the present system, however, such a setup is not necessary, and therefore, we can very simply obtain superconductivity.

\section{The critical current density and critical magnetic field are sufficiently high.}

PNS has an extremely small critical current [1-3], and this fact prevented it from being used in practical applications. In the present superconductivity, however, the critical current is sufficiently high; therefore, we need not be overly concerned by the values of the transport currents. Further, as mentioned, the critical magnetic field in the present superconductivity is very high, which is important when considering transforming a load coil into a superconducting coil. 


\section{It is not necessary to secure extremely low temperatures and high pressures.}

To implement our system, it is not necessary to secure extremely low temperatures or high pressures because the critical current density of our superconductivity is sufficiently large and the temperature is not basically related to the generation of the superconductivity.

\subsection{Important considerations when implementing a superconductor using this system.}

Let us consider the time at which the sample load is disconnected from the system after confirming superconductivity. In this case, does the superconductivity remain? We claim that the superconductivity in the sample should remain having the persistent current $I$ of the toroid. However, when trying to transmit energy through this superconductor, we first need to allow this superconductor to dispose of the energy derived from the self-magnetic fields as a discharge current. This is very important. For example, if we employ only a current source to transmit energy through this superconductor, it cannot discharge a current because of the infinite internal resistance of the current source, which results in normal Ohmic characteristics. In conventional refrigerated superconductors, it is common to employ a current source to transmit energy; however, our superconductor requires additional equipment to discharge the current from the additional self-magnetic field energy. Currently, we are investigating this issue; however, it might be acceptable, when employing only a current source, to secure a pass like relatively small resistance for the discharged current to flow. In this case, however, if we employ an oscilloscope or a portable meter as voltmeter and as a pass of the discharge, they cannot allow the superconductor to discharge a current because their high internal impedances generally prevent the discharge from flowing.

\section{Conclusion}

In this paper, we proposed a new type of superconductivity, in which circuit-approached and temperature-independent properties exist.

As a result of both analytical and numerical calculations, a significantly large critical current density and the Meissner effect, as well as the fact that the sample resistance becomes zero, were found. The significance of our superconductivity is that it can be generated very simply with no refrigeration and with no preparation of specific substances or setups and that it achieves a large critical current density. These properties have not yet been achieved in conventional superconductor studies. As a natural follow-up, we need to confirm this phenomenon in actual experiments. 


\section{Additional information}

This paper is not related to any competing interests such as funding, employment and personal financial interesting. Moreover, this paper is not related to non-financial competing interesting

\section{Acknowledge}

We thank Enago (www.enago.jp) for English language review.

\section{References}

[1] S. Ishiguri, J. Supercond. Nov. Magn. 24, 455 (2011)

[2] S. Ishiguri, Int. J. of Mod. Phys. B, 27, 1350045 (2013)

[3] S. Ishiguri, "New Superconductivity and Theoretical Study on a New Phenomenon of Energy Source with Assistance of Initial Experiments.” Preprints 2018, 2018110636 (2019)

[4] J. Bardeen, L. Cooper, J.R. Schrieffer, Phys. Rev. 108, 1175 (1957)

[5] J. Nagamatsu, et al., Nature 410,63 (2001)

[6] S. Souma, et al., Nature 423, 65 (2003)

[7] P. Sushko, et al., Phys. Rev. Lett. 91, 126401 (2003)

[8] K. Hayashi, et al., J. Am. Chem. Soc.124, 738 (2002)

[9] S. Ishiguri Results in physics, 3, 74 (2013)

[10] M. Ogata, H. Fukuyama, Rep. Prog. Phys., 71, 659 (2008)

[12] M. Azuma, et al., Phys. Rev. Lett, 733463 (1994)

[13] F. C. Zhang, T. M. Rice, Phys. Rev., Vol. B 37, 3759 (1988)

[14] Y. Aharonov, D. Bohm, Phys. Rev. 115, 485 (1959) 